\title{
Systemic therapy as First-Line Treatment for Patients with Metastatic Colorectal Cancer: A Systematic Review and Network meta-analysis of Randomized Clinical Trials
}

Shan Xu ( $\nabla$ alex44_xs@hotmail.com )

Universitat Duisburg-Essen

Ali Sak

Universitat Duisburg-Essen

Yasin Bahadir Erol

Universitat Duisburg-Essen

Research article

Keywords:

Posted Date: January 29th, 2020

DOl: https://doi.org/10.21203/rs.2.22213/v1

License: (c) (1) This work is licensed under a Creative Commons Attribution 4.0 International License.

Read Full License 


\section{Abstract}

Purpose: To assess the relative efficacy and safety of first-line systemic therapies in patients with metastatic CRC ( $\mathrm{mCRC})$.

Experimental Design: A comprehensive literature review was conducted including MEDLINE, Embase, and the Cochrane Central Registry of Controlled Trials for phase II or III Randomized Controlled Trials (RCTs) published up to and including July 15, 2019. We included RCTs in which at least 1 intervention was either a chemotherapeutic agents (such as, fluorouracil, irinotecan, or oxaliplatin) or antibodies targeting angiogenesis (such as, bevacizumab) or agents that act on the epidermal growth factor receptor pathway (such as, cetuximab and panitumumab) or studies reported at least one of the following outcomes: Overall Survival (OS), Progression-Free Survival (PFS) and/or serious adverse events (SAEs). Using a random effect model, we performed a Bayesian network meta-analysis to analyze the probability of optimal therapeutic regime obtained from direct comparisons with indirect evidences. We estimated hazard ratios (HRs) for OS and PFS.

Results: A total of 30 RCTs comprising (12, 146 patients with mCRC comparing 25 different strategies were included. The triple combination FOLFOXIRI [fluorouracil, leucovorin, oxaliplatin, and irinotecan] plus bevacizumab provided significant survival benefits with improved OS over all other treatments. The network meta-analysis also indicated a significant advantage of using FOLFOXIRI plus bevacizumab in comparison to other treatment strategies for PFS. Besides, FOLFOXIRI plus bevacizumab was associated with the lowest risk of serious adverse events.

Conclusions: Our study supported the use of FOLFOXIRI plus bevacizumab as the best first-line regimen and potentially effective and safe strategy for the management of patients with mCRC.

\section{Introduction}

Colorectal cancer (CRC) ranks third amongst all malignant neoplasms and continues to be the leading cause of cancer-associated mortality, worldwide(Hegde et al., 2008). Approximately, $25 \%$ of patients with CRC present with liver metastasis at the initial diagnosis or will develop liver metastasis during the course of their disease(Chow and Chok, 2019). In spite of the emergence of highly effective chemotherapy and advances in surgical techniques, the pool of patients with liver and/or lung-isolated metastasis has expanded and for the majority of patients with metastatic CRC ( $\mathrm{mCRC})$, the treatment remains a clinical challenge (Park et al., 2013). Indeed, for many years 5-fluorouracil (FU)-based regimens has been the backbone of systemic therapy for mCRC. Recent incremental advancements in the systemic therapy for $\mathrm{mCRC}$ have been significantly facilitated with the introduction of several new cytotoxic and biologic agents.

Systemic therapy includes combinations of chemotherapeutic agents (oxaliplatin, irinotecan or fluorouracil) alone or in combination with monoclonal antibodies targeting epidermal growth factor receptor (EGFR; cetuximab and panitumumab) or vascular endothelial growth factor receptor (VEGFR; 
bevacizumab), thereby providing distinctly effective first-line therapeutic regimens for mCRC (Saltz et al., 2004). However, head-to-head randomized trials comparing these therapeutic regimens mentioned above are still lacking, thus there is no evidence to guide optimal regimen for patients $\mathrm{MCRC}$. To overcome these limitations, using a network meta-analysis (NMA) approach, we compared and evaluated the relative therapeutic efficacies of all possible combinations of treatments, by simultaneous integration of direct evidence from head-to-head trials and indirect evidence to rank the different treatments for mCRC.

\section{Method}

\section{Literature Search}

Literature screening was performed according to the method outlined in the Cochrane Handbook for Systematic Reviews of Interventions(Green, 2011). Institutional review board approval was not required. We conducted a comprehensive literature search of electronic databases including MEDLINE, EMBASE, and the Cochrane Central Register of Controlled Trials (Central) databases from inception up to and including July 15, 2019. A combination of MeSH-terms and keywords strategy was applied as follows: "Advance or metastatic colon cancer", "hepatic metastases, liver metastases" "immunotherapy and targeted therapy" (sTable1 in supplement). Also, the references of the selected articles and reviews were manually retrieved to obtain all potentially relevant studies. Retrieved articles were screened and reviewed for their eligibility by two independent reviewers (SX, YBE). Differences in the determination of the study's eligibility were resolved by consensus or through discussion with a third adjudicator (AS). The language of publication was restricted to English.

\section{Study Selection}

We included phase II or III randomized controlled trials (RCT) that met the following inclusion criteria: (a) the study subjects were patients with mCRC; (b) at least 1 of the interventions compared in the trial was either a chemotherapeutic agents (such as, fluorouracil, irinotecan, or oxaliplatin) or antibodies targeting angiogenesis (such as, bevacizumab) or agents that act on the EGFR-related pathway (such as, cetuximab and panitumumab); (c) the primary outcome was overall survival (OS), progression-free survival (PFS), and/or serious adverse events (SAEs) of greater than or equal to grade 3 according to the National Cancer Institute's (NCl) Common Terminology Criteria for Adverse Events (CTCAE). We excluded studies that were not RCTs and had unavailable data.

\section{Data Extraction and Risk of Bias Assessment}

The data extraction from each included study was performed independently by two reviewers (SX, AS) and entered into a standardized, predesigned Microsoft Excel form. The following data were extracted: the first author, the year of publication, country, patient characteristics, treatment strategies, sample size, number of patients evaluated for response, dose, and schedule, median cycles received, and outcomes (median OS and median PFS). For PFS and OS, we extracted the hazard ratio (HR) with a $95 \%$ confidence interval $(95 \% \mathrm{Cl})$ if available. However, when HRs and corresponding Cls were not reported, we estimated 
them by reconstructing individual patient data from published Kaplan- Meier curves with methods described by Guyot and colleagues(Guyot et al., 2012). Authors of included studies were contacted if important data were unclear or not reported. The risk of bias in randomized trials was assessed independently by the reviewers (SX and YBE) using the Cochrane Collaboration tool and the risk-of-bias (RoB 2.0) tool(Higgins and Altman, 2008). Any disagreements were resolved through consensus.

\section{Data Synthesis and Analysis}

We synthesized evidence for 3 outcomes: PFS, OS and any SAEs. For each outcome, we performed a Bayesian NMA using a Markov Chain Monte Carlo (MCMC) simulation technique with 100000 iterations in each of the 3 chains. Noninformative priors (ie, $N[0,10000])$ were chosen for the effect parameters. The analysis was performed under the fixed-effect model, as only 1 trial provided direct evidence for most of the treatment comparisons. However, a random-effects (RE) model was also performed as sensitivity analysis and model fits were compared using deviance information criteria (DIC)(Higgins, 2013). In the comparison of any 2 models, we deemed a better fit model if its DIC was less than the DIC of the other model by at least 5 . Heterogeneity in the network was assessed with the Cochrane Q ( $\chi 2)$ test and quantified using the 12 statistic within each pairwise comparison when 2 or more trials were available for the comparison(Higgins and Thompson, 2002). Because it was uncommon to have both direct and indirect evidence for most comparisons in our networks, we assumed coherence for our analysis (ie, direct and indirect evidence, when both available for a given comparison, were statistically similar). To test the robustness of this assumption we used the node-splitting method to assess whether there was incoherence in any closed loops(Higgins, 2013; Higgins and Thompson, 2002). Relative effects of treatments are reported as HR for survival outcomes (PFS and OS) and odds ratio (OR) for binary outcomes (SAEs) along with corresponding 95\% credible intervals (Crls), the Bayesian equivalent of $95 \%$ Cls. We estimated the overall ranks of treatments by calculating the surface under the cumulative ranking curve (SUCRA) for each (Salanti et al., 2011). The SUCRA index ranges between 0 (or $0 \%$ ) and 1 (or 100\%), where the treatments with highest and lowest SUCRA are considered to be the best and worst treatments, respectively. Network meta-analysis was performed in WinBUGS software (version 1.4.3, MRC Biostatistics Unit) interfacing through R software.

\section{Results}

\section{Overall characteristics of selected studies and Quality of Evidence}

The PRISMA flowchart of included studies is presented in sFigure.1 (shown in supplement). After the exclusion of duplicate studies, a total of 557 records was initially identified through our literature search. After a detailed assessment by the full-text review, 30 trials comprising 12,146 patients with mCRC were included in this meta-analysis (Figure 1). The characteristics of 30 RCTs included in the meta-analysis are summarized in Table 1 The study sample sizes ranged from 25 to 599. These studies were published between 2005 and 2019. The risk of bias and the quality assessment in all studies were presented in supplementary table (sTable 20), which indicated that the quality of the included studies was reliable. 
Moreover, according to the MCMC model, $\mathrm{I}^{2}$ was estimated to be $0.00 \%$. Therefore, there is no heterogeneity in the data, and the results of NMA are stable and reliable.

\section{Overall Survival}

Twenty-five trials comprising 11,175 patients with MCRC comparing 21 treatments were included in the OS analysis (supplementary figure, sFigure 2A). The results indicated that the FOLFOXIRI/Bev treatment strategy was associated with improved OS benefits compared with all other treatments. The key comparison treatments included FOLFOXIRI/Bev vs FOLFOX/Bev with HR, 1.03 (95\% Crl, 0.69-1.52), and FOLFOXIRI/Bev vs FOLFIRI/Bev with HR, 1.07 (95\% Crl, 0.84-1.34). The estimated SUCRA values were 77.2 and $77.1 \%$ for FOLFOXIRI/Bev and FOLFOX/Bev treatment strategies, respectively, suggesting that these two treatment strategies exhibited the highest probability of being the best treatment for improving OS of patients with mCRC (sTable 2A in the Supplement).

\section{Progression-Free Survival}

Twenty-two trials comprising 9,588 patients with $\mathrm{MCRC}$, comparing 25 treatments were included in the PFS analysis (supplementary figure sFigure 2B). FOLFOXIRI/Bev treatment strategy exhibited a significantly higher PFS compared with other strategies. Consistently, the SUCRA analysis also suggested that FOLFOXIRI/Bev treatment strategy was the best treatment in promoting PFS (SUCRA: 93.2\%), followed by FOLFOXIRI (SUCRA: 79.9\%), whereas CapeOX/Cetuximab treatment strategy was least likely to be the optimal treatment strategy in improving PFS (SUCRA: 17.2\%) (Supplementary table, sTable 2B).

\section{Serious Adverse Events}

Eighteen trials comprising 8,986 patients with $\mathrm{mCRC}$, comparing 19 treatment strategies reported adverse events of grade 3 or higher (supplementary figure, sFigure 2C). FOLFOXIRI/Bev treatment strategy was significantly associated with a lower risk of SAEs compared with all other treatments. Consistently, the SUCRA analysis also suggested that FOLFOXIRI/Bev and XELIRI/Bev treatment strategies exhibited the lowest risk of SAEs with SUCRA values of $96.3 \%$ and $83 \%$, respectively. Besides, FOLFOX/Bev/Onartuzumab treatment strategy was associated with a higher risk of SAEs compared with all other treatments (SUCRA values was 4.6\%) (sTable 2C in the Supplement).

\section{Discussion}

Incremental advancements have been made in mCRC therapy ever since the introduction of 5-FU over 40 years ago(Hammond et al., 2016). Moreover, the treatment of $\mathrm{mCRC}$ has been facilitated significantly with the introduction of several new cytotoxic and biologic agents to the 5-FU regimen. Notably, combination regimens that incorporate infusional schedules of 5 -FU in various combinations, including XELOX regimen (oxaliplatin and capecitabine), FOLFOX regimen (leucovorin, 5-FU and oxaliplatin) and FOLFIRI regimen (leucovorin, 5-FU and irinotecan), with or without monoclonal antibody, have significantly improved the clinical outcomes and median overall survival of patients with mCRC. 
In this systematic review and NMA meta-analysis, we estimated the relative efficacy of the different combinations of treatment strategies for outcomes involving OS and PFS in patients with mCRC. Overall survival remains the fundamental endpoint in clinical trials; this meta-analysis found that triple combination FOLFOXIRI [fluorouracil, leucovorin, oxaliplatin, and irinotecan] plus bevacizumab provided significant survival benefits over all the other treatments which were in accord with recent studies(Cremolini et al., 2015). Consistently, a meta-analysis also showed a significant increase in OS with FOLFOXIRI plus bevacizumab in patients with mCRC(Tomasello et al., 2017). Notably, FOLFOXIRI plus bevacizumab was also found to be most effective in promoting PFS. These results are also consistent with the TRIBE study, which indicated significant OS and PFS benefit with the addition of oxaliplatin to FOLFIRI plus bevacizumab in patients with $\mathrm{mCRC}$ (Cremolini et al., 2015). Other recent trials and studies (VISNU-1 trial and QUATTRO Study) have also shown that MCRC patients who received FOLFOXIRI plus bevacizumab exhibited statistically significant improved PFS and OS(Oki et al., 2018; Sastre et al., 2019). Survival benefits needed to be justified against the toxicity of chemotherapy. In the majority of mCRC patients with advanced, systemic treatment remains noncurative, and thus the quality of life becomes a priority. In this meta-analysis, rates of SAEs were high for all treatment strategies except for FOLFOXIRI plus bevacizumab regimen, which exhibited no significant increase in the adverse events compared with other treatments.

Using appropriate statistical approaches, this network meta-analysis is the first study to provide an indirect comparison for the currently available systemic therapy for patients with $\mathrm{mCRC}$, based on all available information from phase II and III randomized trials. This NMA is acknowledged to have several limitations. First, some included studies lacked sufficient comparisons, which may have a certain impact on the result. In addition, the collected results from the included studies were uneven and the sample size of few studies on some drugs was relatively small.

Overall, our study supported the use of FOLFOXIRI-bevacizumab as the best first-line regimen and potentially effective and safe strategy for the management of patients with $\mathrm{mCRC}$. Furthermore, our upto-date analysis provides new insights into existing controversies on systemic therapy for patients with mCRC.

\section{References}

Bendell, J. C., Hochster, H., Hart, L. L., Firdaus, I., Mace, J. R., McFarlane, J. J., Kozloff, M., Catenacci, D., Hsu, J. J., and Hack, S. P. (2017). A Phase II Randomized Trial (G027827) of First-Line FOLFOX Plus Bevacizumab with or Without the MET Inhibitor Onartuzumab in Patients with Metastatic Colorectal Cancer. The oncologist 22, 264-271.

Berlin, J., Bendell, J. C., Hart, L. L., Firdaus, I., Gore, I., Hermann, R. C., Mulcahy, M. F., Zalupski, M. M., Mackey, H. M., and Yauch, R. L. (2013). A randomized phase II trial of vismodegib versus placebo with FOLFOX or FOLFIRI and bevacizumab in patients with previously untreated metastatic colorectal cancer. Clinical Cancer Research 19, 258-267. 
Bokemeyer, C., Bondarenko, I., Hartmann, J., De Braud, F., Schuch, G., Zubel, A., Celik, I., Schlichting, M., and Koralewski, P. (2011). Efficacy according to biomarker status of cetuximab plus FOLFOX-4 as firstline treatment for metastatic colorectal cancer: the OPUS study. Annals of Oncology 22, 1535-1546.

Bokemeyer, C., Bondarenko, I., Makhson, A., Hartmann, J. T., Aparicio, J., De Braud, F., Donea, S., Ludwig, H., Schuch, G., and Stroh, C. (2008). Fluorouracil, leucovorin, and oxaliplatin with and without cetuximab in the first-line treatment of metastatic colorectal cancer. J Clin Oncol.

Cassidy, J., Clarke, S., Diaz-Rubio, E., Scheithauer, W., Figer, A., Wong, R., Koski, S., Rittweger, K., Gilberg, F., and Saltz, L. (2011). XELOX vs FOLFOX-4 as first-line therapy for metastatic colorectal cancer: N016966 updated results. British journal of cancer 105, 58 .

Chow, F. C.-L., and Chok, K. S.-H. (2019). Colorectal liver metastases: An update on multidisciplinary approach. World journal of hepatology 11,150 .

Colucci, G., Gebbia, V., Paoletti, G., Giuliani, F., Caruso, M., Gebbia, N., Cartenì, G., Agostara, B., Pezzella, G., and Manzione, L. (2005). Phase III randomized trial of FOLFIRI versus FOLFOX4 in the treatment of advanced colorectal cancer: a multicenter study of the Gruppo Oncologico Dell'Italia Meridionale. Journal of Clinical Oncology 23, 4866-4875.

Cremolini, C., Loupakis, F., Antoniotti, C., Lupi, C., Sensi, E., Lonardi, S., Mezi, S., Tomasello, G., Ronzoni, M., and Zaniboni, A. (2015). FOLFOXIRI plus bevacizumab versus FOLFIRI plus bevacizumab as first-line treatment of patients with metastatic colorectal cancer: updated overall survival and molecular subgroup analyses of the open-label, phase 3 TRIBE study. The Lancet Oncology 16, 1306-1315.

Díaz-Rubio, E., Tabernero, J., Gómez-Espana, A., Massutí, B., Sastre, J., Chaves, M., Abad, A., Carrato, A., Queralt, B., and Reina, J. J. (2007). Phase III Study of Capecitabine Plus Oxaliplatin Compared With Continuous-Infusion Fluorouracil Plus Oxaliplatin As First-Line Therapy in Metastatic Colorectal Cancer: Final Report of the Spanish. Journal of Clinical Oncology 25, 4224-4230.

Douillard, J. Y., Siena, S., Cassidy, J., Tabernero, J., Burkes, R., Barugel, M., Humblet, Y., Bodoky, G., Cunningham, D., and Jassem, J. (2014). Final results from PRIME: randomized phase III study of panitumumab with FOLFOX4 for first-line treatment of metastatic colorectal cancer. Annals of Oncology $25,1346-1355$.

Ducreux, M., Adenis, A., Pignon, J.-P., François, E., Chauffert, B., Ichanté, J., Boucher, E., Ychou, M., Pierga, J.-Y., and Montoto-Grillot, C. (2013). Efficacy and safety of bevacizumab-based combination regimens in patients with previously untreated metastatic colorectal cancer: final results from a randomised phase II study of bevacizumab plus 5 -fluorouracil, leucovorin plus irinotecan versus bevacizumab plus capecitabine plus irinotecan (FNCLCC ACCORD 13/0503 study). European Journal of Cancer 49, 12361245. 
Ducreux, M., Bennouna, J., Hebbar, M., Ychou, M., Lledo, G., Conroy, T., Adenis, A., Faroux, R., Rebischung, C., and Bergougnoux, L. (2011). Capecitabine plus oxaliplatin (XELOX) versus 5-fluorouracil/leucovorin plus oxaliplatin (FOLFOX-6) as first-line treatment for metastatic colorectal cancer. International journal of cancer $128,682-690$.

Falcone, A., Ricci, S., Brunetti, I., Pfanner, E., Allegrini, G., Barbara, C., Crino, L., Benedetti, G., Evangelista, W., and Fanchini, L. (2007). Phase III trial of infusional fluorouracil, leucovorin, oxaliplatin, and irinotecan (FOLFOXIRI) compared with infusional fluorouracil, leucovorin, and irinotecan (FOLFIRI) as first-line treatment for metastatic colorectal cancer: the Gruppo Oncologico Nord Ovest. Journal of Clinical Oncology 25, 1670-1676.

Folprecht, G., Gruenberger, T., Bechstein, W., Raab, H.-R., Weitz, J., Lordick, F., Hartmann, J., StoehlmacherWilliams, J., Lang, H., and Trarbach, T. (2014). Survival of patients with initially unresectable colorectal liver metastases treated with FOLFOX/cetuximab or FOLFIRI/cetuximab in a multidisciplinary concept (CELIM study). Annals of Oncology 25, 1018-1025.

Fuchs, C. S., Marshall, J., Mitchell, E., Wierzbicki, R., Ganju, V., Jeffery, M., Schulz, J., Richards, D., SoufiMahjoubi, R., and Wang, B. (2007). Randomized, controlled trial of irinotecan plus infusional, bolus, or oral fluoropyrimidines in first-line treatment of metastatic colorectal cancer: results from the BICC-C Study. Journal of Clinical Oncology 25, 4779-4786.

García-Carbonero, R., van Cutsem, E., Rivera, F., Jassem, J., Gore, I., Tebbutt, N., Braiteh, F., Argiles, G., Wainberg, Z. A., and Funke, R. (2017). Randomized phase II trial of parsatuzumab (anti-EGFL7) or placebo in combination with FOLFOX and bevacizumab for first-line metastatic colorectal cancer. The oncologist 22, 375-e330.

Giantonio, B. J., Catalano, P. J., Meropol, N. J., O'Dwyer, P. J., Mitchell, E. P., Alberts, S. R., Schwartz, M. A., and Benson, A. r. (2007). Bevacizumab in combination with oxaliplatin, fluorouracil, and leucovorin (FOLFOX4) for previously treated metastatic colorectal cancer: results from the Eastern Cooperative Oncology Group Study E3200. Journal of Clinical Oncology 25, 1539.

Giuliani, F., Vita, F. D., Maiello, E., Nugnes, I., Bisceglie, M. D., Romito, S., Lorusso, V., Catalano, G., Agostara, B., and Colucci, G. Folfiri versus xeliri in untreated advanced colorectal cancer: A phase II randomised trial of the Gruppo Oncologico dell' Italia Meridionale (prot. GOIM 2405). 6, 120-121.

Green, S. (2011). Cochrane handbook for systematic reviews of interventions version 5.1. 0 [updated March 2011]. The Cochrane Collaboration.

Guyot, P., Ades, A., Ouwens, M. J., and Welton, N. J. (2012). Enhanced secondary analysis of survival data: reconstructing the data from published Kaplan-Meier survival curves. BMC medical research methodology 12,9 . 
Hammond, W. A., Swaika, A., and Mody, K. (2016). Pharmacologic resistance in colorectal cancer: a review. Therapeutic advances in medical oncology $8,57-84$.

Hegde, S. R., Sun, W., and Lynch, J. P. (2008). Systemic and targeted therapy for advanced colon cancer. Expert review of gastroenterology \& hepatology 2, 135-149.

Heinemann, V., von Weikersthal, L. F., Decker, T., Kiani, A., Vehling-Kaiser, U., Al-Batran, S.-E., Heintges, T., Lerchenmüller, C., Kahl, C., and Seipelt, G. (2014). FOLFIRI plus cetuximab versus FOLFIRI plus bevacizumab as first-line treatment for patients with metastatic colorectal cancer (FIRE-3): a randomised, open-label, phase 3 trial. The lancet oncology 15, 1065-1075.

Higgins, J. (2013). Identifying and addressing inconsistency in network meta-analysis. Cochrane comparing multiple interventions methods group Oxford training event 2013.

Higgins, J. P., and Altman, D. G. (2008). Assessing risk of bias in included studies. Cochrane handbook for systematic reviews of interventions: Cochrane book series, 187-241.

Higgins, J. P., and Thompson, S. G. (2002). Quantifying heterogeneity in a meta-analysis. Statistics in medicine 21, 1539-1558.

Hochster, H. S., Hart, L. L., Ramanathan, R. K., Childs, B. H., Hainsworth, J. D., Cohn, A. L., Wong, L., Fehrenbacher, L., Abubakr, Y., and Saif, M. W. (2008). Safety and efficacy of oxaliplatin and fluoropyrimidine regimens with or without bevacizumab as first-line treatment of metastatic colorectal cancer: results of the TREE Study. Journal of Clinical Oncology 26, 3523-3529.

Hurwitz, H. I., Tan, B. R., Reeves, J. A., Xiong, H., Somer, B., Lenz, H. J., Hochster, H. S., Scappaticci, F., Palma, J. F., and Price, R. (2019). Phase II Randomized Trial of Sequential or Concurrent FOLFOXIRIBevacizumab Versus FOLFOX-Bevacizumab for Metastatic Colorectal Cancer (STEAM). The oncologist 24, 921-932.

Infante, J. R., Reid, T. R., Cohn, A. L., Edenfield, W. J., Cescon, T. P., Hamm, J. T., Malik, I. A., Rado, T. A., McGee, P. J., and Richards, D. A. (2013). Axitinib and/or bevacizumab with modified FOLFOX-6 as firstline therapy for metastatic colorectal cancer: A randomized phase 2 study. Cancer 119, 2555-2563.

Kim, S. T., Hong, Y. S., Lim, H. Y., Lee, J., Kim, T. W., Kim, K.-P., Kim, S. Y., Baek, J. Y., Kim, J. H., and Lee, K.W. (2014). S-1 plus oxaliplatin versus capecitabine plus oxaliplatin for the first-line treatment of patients with metastatic colorectal cancer: updated results from a phase 3 trial. BMC cancer 14,883 .

Loupakis, F., Cremolini, C., Masi, G., Lonardi, S., Zagonel, V., Salvatore, L., Cortesi, E., Tomasello, G., Ronzoni, M., and Spadi, R. (2014). Initial therapy with FOLFOXIRI and bevacizumab for metastatic colorectal cancer. New England Journal of Medicine 371, 1609-1618.

Oki, E., Kato, T., Bando, H., Yoshino, T., Muro, K., Taniguchi, H., Kagawa, Y., Yamazaki, K., Yamaguchi, T., and Tsuji, A. (2018). A multicenter clinical phase II study of FOLFOXIRI plus bevacizumab as first-line 
therapy in patients with metastatic colorectal cancer: QUATTRO study. Clinical colorectal cancer 17, 147155.

Park, J., Kim, T., Lee, K., Han, S., Oh, D., Im, S., Kang, G., Chie, E., Ha, S., and Jeong, S. (2013). The beneficial effect of palliative resection in metastatic colorectal cancer. British journal of cancer 108, 1425 .

Pectasides, D., Papaxoinis, G., Kalogeras, K. T., Eleftheraki, A. G., Xanthakis, I., Makatsoris, T., Samantas, E., Varthalitis, I., Papakostas, P., and Nikitas, N. (2012). XELIRI-bevacizumab versus FOLFIRI-bevacizumab as first-line treatment in patients with metastatic colorectal cancer: a Hellenic Cooperative Oncology Group phase III trial with collateral biomarker analysis. BMC cancer 12, 271.

Porschen, R., Arkenau, H.-T., Kubicka, S., Greil, R., Seufferlein, T., Freier, W., Kretzschmar, A., Graeven, U., Grothey, A., and Hinke, A. (2007). Phase III study of capecitabine plus oxaliplatin compared with fluorouracil and leucovorin plus oxaliplatin in metastatic colorectal cancer: a final report of the AIO Colorectal Study Group. Journal of Clinical Oncology 25, 4217-4223.

Salanti, G., Ades, A., and loannidis, J. P. (2011). Graphical methods and numerical summaries for presenting results from multiple-treatment meta-analysis: an overview and tutorial. Journal of clinical epidemiology 64, 163-171.

Saltz, L. B., Meropol, N. J., Loehrer Sr, P. J., Needle, M. N., Kopit, J., and Mayer, R. J. (2004). Phase II trial of cetuximab in patients with refractory colorectal cancer that expresses the epidermal growth factor receptor. Journal of clinical oncology 22, 1201-1208.

Sastre, J., Vieitez, J. M., Gomez-España, M. A., Gil Calle, S., Salud Salvia, A., Suárez, B. G., Garcia-Alfonso, P., Martinez de Castro, E., Quintero Aldana, G. A., and Reina-Zoilo, J. J. (2019). Randomized phase III study comparing FOLFOX+ bevacizumab versus folfoxiri+ bevacizumab (BEV) as 1st line treatment in patients with metastatic colorectal cancer ( $\mathrm{mCRC}$ ) with $\geq 3$ baseline circulating tumor cells (bCTCs). In, (American Society of Clinical Oncology).

Soda, H., Maeda, H., Hasegawa, J., Takahashi, T., Hazama, S., Fukunaga, M., Kono, E., Kotaka, M., Sakamoto, J., and Nagata, N. (2015). Multicenter Phase II study of FOLFOX or biweekly XELOX and Erbitux (cetuximab) as first-line therapy in patients with wild-type KRAS/BRAF metastatic colorectal cancer: The FLEET study. BMC cancer 15, 695.

Souglakos, J., Androulakis, N., Syrigos, K., Polyzos, A., Ziras, N., Athanasiadis, A., Kakolyris, S., Tsousis, S., Kouroussis, C., and Vamvakas, L. (2006). FOLFOXIRI (folinic acid, 5-fluorouracil, oxaliplatin and irinotecan) vs FOLFIRI (folinic acid, 5-fluorouracil and irinotecan) as first-line treatment in metastatic colorectal cancer (MCC): a multicentre randomised phase III trial from the Hellenic Oncology Research Group (HORG). British journal of cancer 94, 798.

Souglakos, J., Ziras, N., Kakolyris, S., Boukovinas, I., Kentepozidis, N., Makrantonakis, P., Xynogalos, S., Christophyllakis, C., Kouroussis, C., and Vamvakas, L. (2012). Randomised phase-II trial of CAPIRI 
(capecitabine, irinotecan) plus bevacizumab vs FOLFIRI (folinic acid, 5-fluorouracil, irinotecan) plus bevacizumab as first-line treatment of patients with unresectable/metastatic colorectal cancer (mCRC). British journal of cancer 106, 453.

Tol, J., Koopman, M., Cats, A., Rodenburg, C. J., Creemers, G. J., Schrama, J. G., Erdkamp, F. L., Vos, A. H., Van Groeningen, C. J., and Sinnige, H. A. (2009). Chemotherapy, bevacizumab, and cetuximab in metastatic colorectal cancer. New England Journal of Medicine 360, 563-572.

Tomasello, G., Petrelli, F., Ghidini, M., Russo, A., Passalacqua, R., and Barni, S. (2017). FOLFOXIRI plus bevacizumab as conversion therapy for patients with initially unresectable metastatic colorectal cancer: a systematic review and pooled analysis. JAMA oncology 3, e170278-e170278.

Van Cutsem, E., Kohne, C.-H., Láng, I., Folprecht, G., Nowacki, M. P., Cascinu, S., Shchepotin, I., Maurel, J., Cunningham, D., and Tejpar, S. (2011). Cetuximab plus irinotecan, fluorouracil, and leucovorin as first-line treatment for metastatic colorectal cancer: updated analysis of overall survival according to tumor KRAS and BRAF mutation status. J clin Oncol 29, 2011-2019.

\section{Table}

Table1: Study and patient population characteristics of included studies 
Author/Years

Sample Intervention

size (n)

Hochster, 2008(Hochster et al., 2008)

71
FOLFOX+ BEV

64

Median

age

$4 \quad$ OS

SAE

72
49
48

Cremolini, 2015(Cremolini et al., 2015)

256 FOLFIRI + BEV

CapeOx + BEV

FOLFOX

CapeOX

62.5

62

62

60

OS

PFS

252

FOLFOXIRI + BEV

144

FOLFIRI

60.5

Fuchs, 2007 (Fuchs et al., 2007)

Fol

57

FOLFIRI + BEV

72

Ducreux, 2013(Ducreux et al., 2013)

Ducreux, 2013(Ducreux et at., 2013)

73

Pectasides, 2012 (Pectasides et al., 2012)

143

73

143

XELIRI +BEV

$\begin{array}{ll}142 & \text { FOLFIRI +BEV } \\ 286 & \text { FOLFOX + BEV } \\ & \\ \end{array}$
Giantonio, 2007 (Giantonio et al.,
2007)

Giantonio, 2007 (Giantonio etal.

Cutsem, 2011(Van Cutsem et al.,
2011)

\begin{tabular}{llll}
291 & FOLFOX & 60 & \\
\hline 243 & BEV & 59.6 & \\
\hline 599 & FOLFIRI + Cetuximab & 61 & OS \\
& & & PFS \\
& & & SAE \\
599 & FOLFIRI & 61 & \\
& & & \\
& &
\end{tabular}


Bokemeyer, 2008 (Bokemeyer et al., 2008)

169

FOLFOX + Cetuximab

62

PFS

SAE

Tol, 2009 (Tol et al., 2009) 2014)
168

368

FOLFOX

CapeOx + BEV
60

62

OS

PFS

SAE

62

Cetuximab

593

FOLFOX +

Panitumumab
62.5

OS

PFS

SAE

61

66

OS

Souglakos, 2006 (Souglakos et al., 146

590

FOLFOX

FOLFIRI

2006)

137

FOLFOXIRI

66

Falcone, 2007 (Falcone et al., 2007)

122

FOLFOXIRI

64

OS

PFS

SAE

122

FOLFIRI

62

Colucci, 2005 (Colucci et al., 2005)

178

FOLFIRI

62

os

SAE

182

FOLFOX

62

Díaz-Rubio,2007 (Díaz-Rubio et al., 2007)

171

CapeOX

64

OS

171

FUOX

65 
Porschen, 2007 (Porschen et al., 2007)
$241 \quad$ CapeOX

233

156

Ducreux, 2011 (Ducreux et al., 2011)

Cassidy, 2011 (Cassidy et al., 2011)

Bokemeyer, 2011 (Bokemeyer et al., 2011)

FUFOX

CapeOX

150

317

317

349

350

168

FOLFOX

CapeOX

FOLFOX
FOLFOX

62

OS

SAE

349

64

66

OS

PFS

61

FOLFOX +BEV

60

61

CapeOX + BEV

60

OS

PFS

SAE

169 FOLFOX + Cetuximab 62

Heinemann, 2014 (Heinemann et al., 2014)

297

FOLFIRI + Cetuximab

64

OS

PFS

SAE

295

FOLFIRI +BEV

65

Infante, 2013(Infante et al., 2013)

39

FOLFOX + Axitinib

61

OS

PFS

SAE

$\begin{array}{lll}43 & \text { FOLFOX + BEV } & 64 \\ 41 & \begin{array}{l}\text { FOLFOX + BEV + } \\ \text { Axitinib }\end{array} & 59\end{array}$

Bendell, 2017 (Bendell et al., 2017)
FOLFOX +BEV
+Onartuzumab

60

OS

PFS 


$\begin{array}{lclcl} & 97 & \text { FOLFOX + BEV } & 62 & \\ \begin{array}{l}\text { Carbonero, 2017 (García-Carbonero } \\ \text { et al., 2017) }\end{array} & 63 & \text { FOLFOX+ BEV + } & 62 & \text { OS } \\ \text { Parsatuzumab } & & \text { PFS }\end{array}$

$62 \quad$ FOLFOX + BEV 62

Kim, 2014 (Kim et al., 2014)

172 CapeOX

62

OS

PFS

\begin{tabular}{|c|c|c|c|c|}
\hline & 168 & CapeOX +S-1 + SOX & 61 & \\
\hline \multirow{4}{*}{$\begin{array}{l}\text { Loupakis, } 2014 \text { (Loupakis et al., } \\
\text { 2014) }\end{array}$} & 256 & FOLFIRI + BEV & 60 & OS \\
\hline & & & & PFS \\
\hline & & & & SAE \\
\hline & 252 & FOLFOXIRI + BEV & 60.5 & \\
\hline
\end{tabular}

Souglakos, 2012 (Souglakos et al., 2012)

159 XELIRI + BEV

PFS

SAE

\begin{tabular}{|c|c|c|c|c|}
\hline & 160 & FOLFIRI + BEV & - & \\
\hline \multirow{2}{*}{$\begin{array}{l}\text { Folprecht, } 2014 \text { (Folprecht et al., } \\
\text { 2014) }\end{array}$} & 56 & FOLFOX + Cetuximab & - & os \\
\hline & 55 & FOLFIRI + Cetuximab & - & PFS \\
\hline \multirow[t]{4}{*}{ Hurwitz, 2019 (Hurwitz et al., 2019) } & 95 & FOLFOX + BEV & 58 & OS \\
\hline & & & & PFS \\
\hline & & & & SAE \\
\hline & 92 & FOLFOXIRI + BEV & 58 & \\
\hline \multirow[t]{2}{*}{ Giuliani, 2008 (Giuliani et al.) } & 20 & FOLFIRI & - & PFS \\
\hline & 34 & XELIRI & - & \\
\hline \multirow[t]{5}{*}{ Berlin, 2013 (Berlin et al., 2013) } & 64 & FOLFOX + BEV & & PFS \\
\hline & 60 & $\begin{array}{l}\text { FOLFOX + BEV } \\
\text { +Vismodegib }\end{array}$ & & SAE \\
\hline & 37 & FOLFIRI + BEV & & \\
\hline & 38 & FOLFIRI + BEV + & & \\
\hline & \multicolumn{2}{|c|}{ Page $15 / 18$} & & \\
\hline
\end{tabular}




\section{Vismodegib}

Soda, 2015 (Soda et al., 2015)

XELIRI : CAPIRI, Irinotecan plus capecitabine, FOLFIRI: irinotecan plus fluorouracil plus leucovorin; BEV: bevacizumab; SOX: oxaliplatin; FUOX: high-dose fluorouracil plus oxaliplatin; FUFOX: fluorouracil plus folinic acid plus oxaliplatin; FOLFOX fluorouracil and leucovorin with oxaliplatin. CapeOX: XELOX, capecitabine plus oxaliplatin.

\section{Figures}

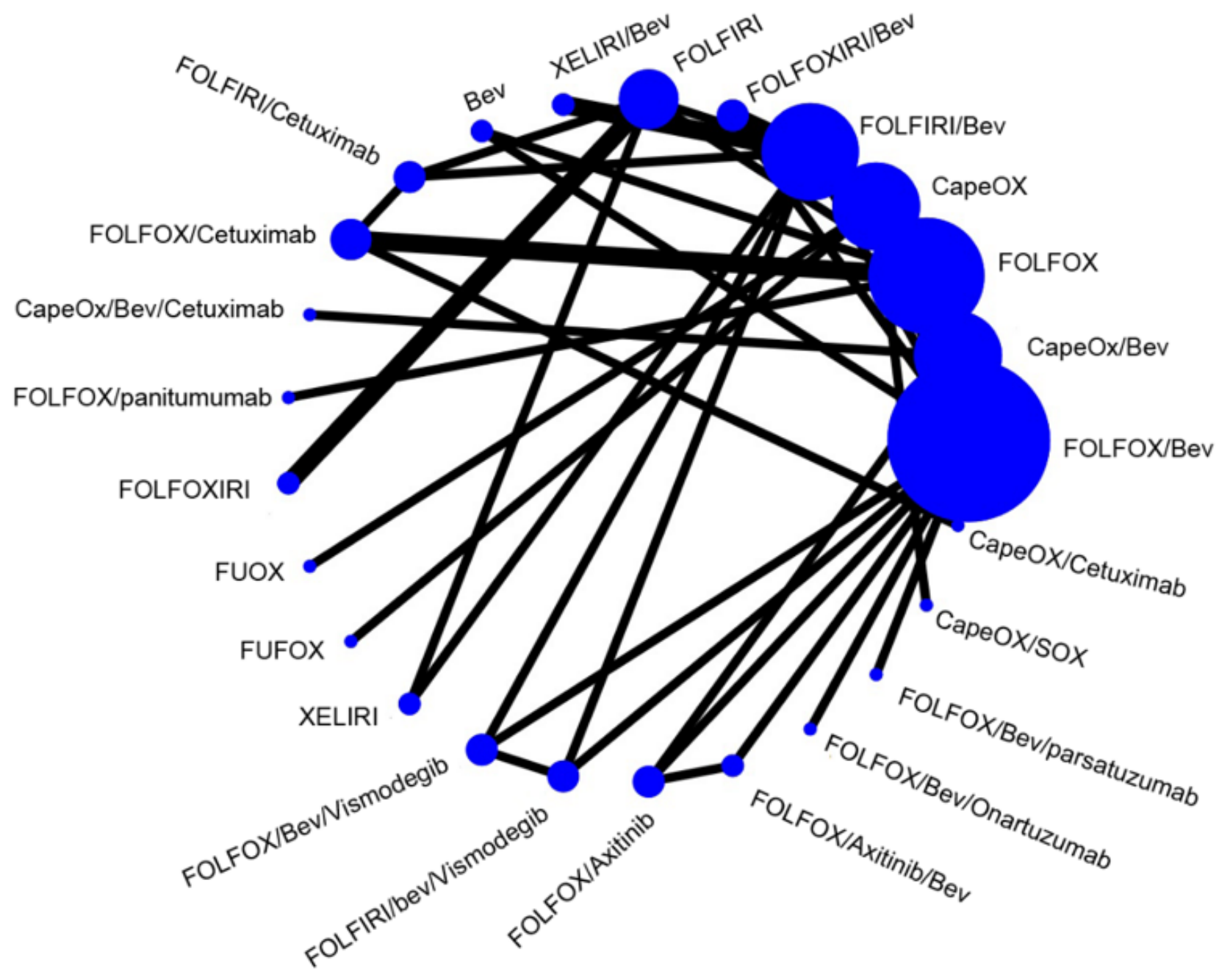

\section{Figure 1}

Network of the comparisons for the Bayesian network meta-analysis. XELIRI : CAPIRI, Irinotecan plus capecitabine , FOLFIRI: irinotecan plus fluorouracil plus leucovorin; BEV: bevacizumab; SOX: oxaliplatin; 
FUOX: high-dose fluorouracil plus oxaliplatin; FUFOX: fluorouracil plus folinic acid plus oxaliplatin; FOLFOX fluorouracil and leucovorin with oxaliplatin. CapeOX: XELOX, capecitabine plus oxaliplatin.

\section{A: Overall survival}

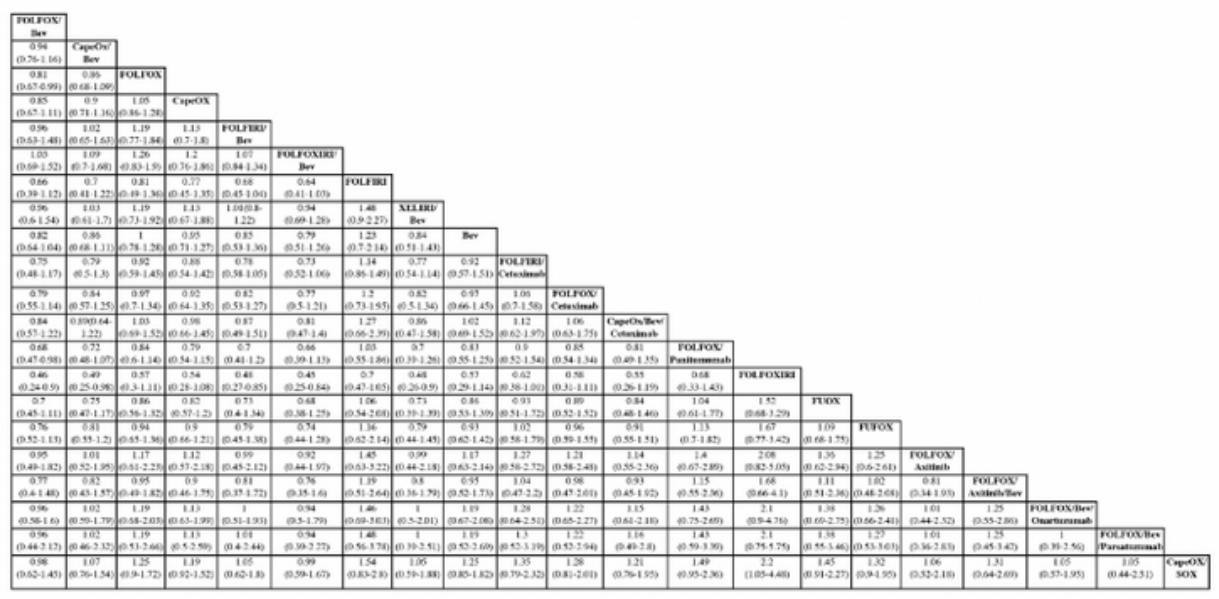

\section{B: Progression-free survival}

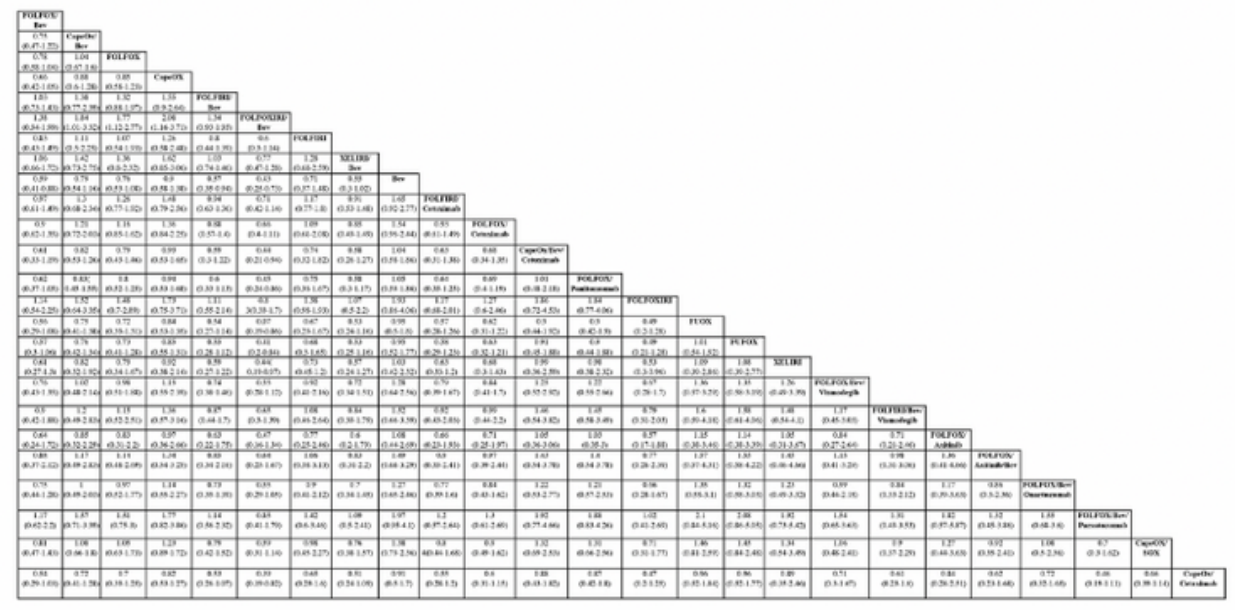

\section{C: Serious Adverse Events}

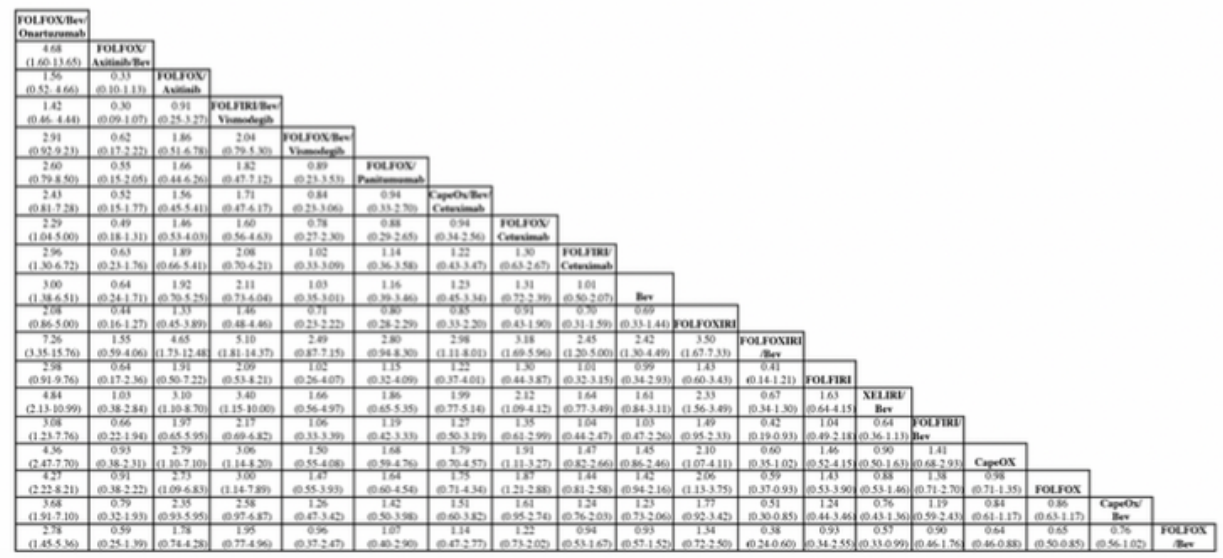

\section{Figure 2}

Pooled Estimates for All Possible Treatment Effects for Each Outcome. XELIRI : CAPIRI, Irinotecan plus capecitabine , FOLFIRI: irinotecan plus fluorouracil plus leucovorin; BEV: bevacizumab; SOX: oxaliplatin; 
FUOX: high-dose fluorouracil plus oxaliplatin; FUFOX: fluorouracil plus folinic acid plus oxaliplatin; FOLFOX fluorouracil and leucovorin with oxaliplatin. CapeOX: XELOX, capecitabine plus oxaliplatin.

\section{Supplementary Files}

This is a list of supplementary files associated with this preprint. Click to download.

- Supplement12.03.docx

- PRISMA2009checklist.doc 